\title{
A Smart Approach for Delivery of Nanosized Phenytoin using Biomaterial Isolated from Fragaria ananassa
}

\author{
Sushant Kumar ${ }^{1, *}$, Nookala Venkala Satheesh Madhav², Anurag Verma ${ }^{3}$, Kamla Pathak ${ }^{1}$ \\ 'Faculty of Pharmacy, Pharmacy College Saifai, Uttar Pradesh University of Medical Sciences, Saifai, Etawah, Uttar Pradesh, INDIA. \\ ${ }^{2}$ Faculty of Pharmacy, DIT Univesity, Dehradun, Uttarakhand, INDIA. \\ ${ }^{3}$ School of Pharmaceutical Science, IFTM University, Moradabad, Uttar Pradesh, INDIA.
}

\begin{abstract}
Objectives: The bionanosuspension was prepared by using the isolated biopolymer from Fragaria ananassa fruit which serve as a potential, natural polymeric nature biomaterial. The isolated biomaterial was used for the preparation of nanosized phenytoin loaded bionanoparticles in the form of bionanosuspension. The isolated biopolymer was characterized for its polymeric properties and its potentiality was evaluated in the delivery of nanosized phenytoin. Materials and Methods: The isolated biopolymer from Fragaria ananassa was used for the formulation of phenytoin loaded bionanosuspension. The sonication method was used for the preparation of bionanosuspension having bionanoparticles in nano range loaded with nanosized phenytoin. The accurate quantity of phenytoin and isolated biopolymer was mixed with other excipients like dextrose as nanosizent, polyvinyl alcohol, sodium benzoate with double distilled water and then sonicated in bath sonicator for 15 cycles $(1$ cycle $=3 \mathrm{~min})$. Results: The isolated biopolymer was characterized for DSC, FTIR, NMR, Mass and Zeta particle size analysis. The obtained results confirm its polymeric nature in different analysis. The prepared bionanoparticles showed the release of phenytoin in sustained manner over $36 \mathrm{hr}$. The release kinetic study was done by using the BIT-SOFT 1.12 software and $t 50 \%$ and $t 80 \%, r^{2}$ were
\end{abstract}

calculated. The formulation PF4 was found to be the best formulation having $t 50 \%$ of $17 \mathrm{hr}$ and $\mathrm{t} 80 \%$ of $28 \mathrm{hr}$ with $r^{2}$ value of 0.9912 . The best formulation PF4 showed up to $90.64 \%$ drug release over $36 \mathrm{hr}$. According to the release kinetic study the best fit model was found to be KorsmeyerPeppas and the mechanism of drug release was found to be anomalous transport. Conclusion: Thus the isolated biopolymer from fruit pulp of Fragaria ananassa can be safely used for the preparation of nanosized phenytoin loaded bionanoparticles in the delivery of phenytoin in significant amount for the treatment of epilepsy.

Key words: Biopolymer, Bionanoparticles, Bionanosuspension, Phenytoin, Epilepsy, Nanosizing, Fragaria ananassa.

\section{Correspondence}

Mr. Sushant Kumar

Faculty of Pharmacy, Pharmacy College Saifai, Uttar Pradesh University of Medical Sciences, Saifai, Etawah-206130, Uttar Pradesh, INDIA.

Phone no: +917376766071

Email: k.sushant25@gmail.com

DOI: 10.5330/ijpi.2020.3.55

\section{INTRODUCTION}

Polymers are the first choice for designing of novel drug delivery systems for sustained release, controlled release, extended release and targeted drug delivery systems. ${ }^{1}$ These synthetic and semisysnthetic polymers are prepared by a number of chemical treatment and purification processes which may produce adverse effects on human physiological systems. These polymers are not biodegradable and not biocompatible completely and also are very costly to produce at industrial level.

Now days the researches has proved that the biopolymers are one of the most novel and intelligent biomaterial which are isolated from the various natural sources like bark, seeds, flowers, fruits pulps, leaves and other natural sources. ${ }^{2}$ They have a number of novel properties which may be used as an alternative to synthetic and semisynthetic polymers. The isolated biopolymer is biodegradable and biocompatible in nature. Since these are isolated from the natural sources and their excellent biocompatibility these biopolymers may be used as a biocarrier for drug delivery. As this biopolymer have good film forming ability, mucoadhesivity, sustainability, retardability, release rate controlling ability these are the excellent biomaterial. ${ }^{3}$ These natural biopolymer having significant polymeric properties may be used for designing of the novel drug and targeted drug delivery systems. ${ }^{4}$

Epilepsy is a neurological disorder in which the brain condition becomes abnormal and shows the repeated, uncontrolled and sudden changes in brain. In epilepsy there are the abnormal changes in electrical activity of brain. The epileptic episodes are called as convulsions. There are two types of seizures one is generalized seizure and other is partial seizure. The generalized seizure occurs in whole brain and the partial seizure occurs in any one part of the brain. The partial seizure can be converted in the generalized seizure.

In this research work bionanosuspension having the bionanoparticles loaded with nanosized phenytoin has been prepared by using the novel biomaterial from fruit pulp of Fragaria ananassa. ${ }^{5}$ The potentiality of biopolymers was evaluated for developing bionanoparticles and its suitability for release of phenytoin.

\section{MATERIALS AND METHODS}

\section{Materials}

Phenytoin was obtained as a gift sample from Affy pharma private limited, Baddi. The Fragaria ananassa (Strawberry) fruit was purchased from the market of Lucknow. All other chemicals used were of analytical grade.

\section{Methods \\ Isolation of Biopolymer}

The Fragaria ananassa fruit was purchased from the local market of Lucknow. 500gram of fruit pulp was weighed and washed with distilled water. The weighed fruit pulp was taken and grinded in grinder as a paste. 
If necessary small quantity of distilled water may be added during the grinding. This paste was filtered through the muslin cloth. The collected filtrate was centrifuge at $5000 \mathrm{rpm}$ for $10 \mathrm{~min} .{ }^{6}$ After centrifugation the supernatant was taken. Centrifugation was done to remove any residue. Then half of the supernatant was treated with methanol in 1:1ratio. These were placed in refrigerator for overnight. Then after treatment these were centrifuged at $5000 \mathrm{rpm}$ for $30 \mathrm{~min}$. The supernatant was discarded and the biomaterial as a sediment collected and air dried. If any moisture is there, can be dried in desiccators for $48 \mathrm{hr}$. If the biomaterial consists of any oil, can be removed by washing with acetone or chloroform. This procedure was repeated and optimized for six times and the yield was calculated. The obtained biomaterial was passed through sieve number 200 and stored for further use. ${ }^{7}$

\section{Characterization of Isolated Biopolymer}

The physico-chemical properties of isolated biopolymer were characterized for color, odor, taste and solubility. The chemical tests for presence of carbohydrate, starch and proteins were also performed. The isolated biopolymer was also characterized for SEM analysis, DSC testing, IR spectroscopy, mass spectroscopy and NMR spectroscopy.

\section{Nanosizing of Phenytoin}

$500 \mathrm{mg}$ of phenytoin was taken and dissolved in $25 \mathrm{ml}$ of methanol. The clear solution was sonicated for 15 cycles continuously. During sonication $25 \mathrm{ml}$ of purified water as added slowly drop by drop till precipitation was observed. The obtained precipitate was allowed for centrifugation. After each sonication cycle the sample was allowed for absorbance and $\%$ transmittance $(\% \mathrm{~T})$ and \% blockage (100-\%transmittance) measurement. The residue was recovered and then dried to collect the nanosized phenytoin in nanoparticles range. This nanosized phenytoin obtained by this standard solvent evaporation method, was evaluated for different parameters. The dried nanosized phenytoin was packed and stored for further use. The phenytoin was also nanosized by novel sonication method. Here 500mg of phenytoin was taken and mixed with dextrose and $25 \mathrm{ml}$ double distilled water. This dispersed solution was sonicated for 15 cycles continuously. During sonication $25 \mathrm{ml}$ of purified water as added slowly drop by drop till precipitation was observed. ${ }^{6}$ The obtained precipitate was allowed for centrifugation. After each sonication cycle the sample was allowed for absorbance and \% transmittance $(\% \mathrm{~T})$ and $\%$ blockage (100-\%transmittance) measurement. The residue was recovered and then dried to collect the nanosized free flowing phenytoin powder. The procedure was repeated in triplicate.

\section{Drug-Excipient Interaction Study}

The drug-biopolymer interaction study was performed by the U.V. spectroscopy method. The phenytoin -biopolymer mixture was prepared in ratio of $1: 1,1: 3$ and 3:1 by wet and dry mixing. After mixing the drug and polymer mixtures were stored at $50^{\circ} \mathrm{C}$ for three days in wet method and then the mixture was diluted with solvent and scanned for the absorption maxima $\left(\lambda_{\max }\right)$. In dry method the three different ratio of drug-biopolymer was prepared in their physical form and then after storage at room temperature this was diluted with $2 \mathrm{ml}$ of methanol and then scanned by UV spectrophotometer for any change in $\lambda_{\max }$.

\section{Formulation of Phenytoin Loaded Bionanosuspension}

The formulations of bionanosuspension were prepared by using different drug -biopolymer ratio. The drug- biopolymer ratio is given in the Table 1. The bionanosuspension was prepared by sonication of the mixture of drug and biopolymer along with other excipients like polyvinyl alcohol as suspending agent, sodium benzoate as the preservative, purified water and dextrose as nanosizent. The phenytoin, Fragaria ananassa biopolymer and other excipients were accurately weighed and triturated with addition of the double distilled water. This mixture was sonicated for 3 cycles. Then $0.5 \mathrm{ml}$ of $0.5 \%$ polyvinyl alcohol was added during sonication. The volume was made up to $10 \mathrm{ml}$ with double distilled water having sodium benzoate $0.1-0.5 \%$. Add dextrose if necessary as nanosizing agent and allowed for sonication for 15 cycles. After sonication the bionanosuspension was refrigerated for two days. If no settlement is there then it means the formulation is optimized. If settlement is there, $0.5 \mathrm{ml}$ of $0.5 \%$ polyvinyl alcohol was again added and allowed for sonication for 10 cycles and refrigerated for $48 \mathrm{hr}$. The different formulations were prepared and after optimization according to stability the formulations PF1 - PF8 were prepared. After formulation their stability was tested and then evaluated for different parameters including release study.

\section{Characterization of Phenytoin Loaded Bionanosuspension}

\section{Dispersiblity Study of Bionanosuspension}

$10 \mathrm{ml}$ of the formulated bionanosuspension was taken and dispersed in $20 \mathrm{ml}$ of the distilled water in a test tube. The time for settling of the dispersed nanoparticles in the bottom was noted and then again the nanoparticles was redispersed and noticed for the redispersion. After shaking any lump or aggregates or any precipitation formation was observed. The procedure was repeated in triplicate.

\section{pH Study of Bionanosuspension}

The ph of formulated bionanosuspension was evaluated with digital $\mathrm{pH}$ meter. The study was done in triplicate and the mean was taken and checked that the $\mathrm{pH}$ of the nanosuspension is in required range or not. The procedure was repeated in triplicate.

\section{\% Entrapment Efficacy of Loaded Bionanosuspension}

The freshly formulated bionanosuspension was taken and centrifuged at 5000rpm in ultracentrifuge .After centrifugation the supernatant was taken and diluted up to $10 \mu \mathrm{g} / \mathrm{ml}$ and the amount of drug unincorporated was measured by determining the absorbance under UV spectroscopy at $216 \mathrm{~nm}$. The amount of the drug loaded in nanoparticles was calculated by subtracting the amount of free drug in supernatant from the initial amount of drug taken in formulation. The procedure was repeated in triplicate. This determination was done in triplicate and average was calculated by using the following formula:

Amount of the drug loaded in nanoparticles

\%Entrapment efficacy $=\frac{}{\text { Initial amount of the drug taken in formulation }} \times 100$

\section{Particle Size screening of the Nanoparticles in Bionanosuspension by UV Method}

The bionanosuspension was evaluated by measuring the \%transmittance of the bionanosuspension. The \%transmittance was measured as a function of the particle size in nano range done by sonication method. The $\%$ transmittance depends on the particle size range at the particular range that define the size particles are below range and size of the particles beyond the range required. The \% transmittance was determined before and after the sonication cycle. The $\%$ transmittance at different wavelength indicates that when the light is passed through the particles means the particle size is below that wavelength which indicates that $\%$ of the particles is below $400 \mathrm{~nm}$ in the mixture and the \% blockade shows that the $\%$ of particles is above $400 \mathrm{~nm}$. The $\%$ transmittance was measured by using the UV spectrophotometer. After each sonication cycle the $\%$ transmittance was found to be increased due to reduction of the particles to nano range. The effect of sonication on $\%$ transmittance 
was observed after sonication and measuring the \% transmittance after each sonication cycle. The procedure was repeated in triplicate.

\section{Particle Size Analysis}

The particle size of the bionanosuspension was studied by characterizing with the Malvern zetasizer. The particle size distribution by intensity was confirmed by using the zetasizer.

\section{In vitro release study of bionanosuspension}

The in vitro release study was performed for the all formulation. In vitro release study was performed by novel static method by using modified M.S. Diffusion apparatus. It consists of two compartment one donor and one receiver compartment. The formulation for release study was taken in donor compartment $(1 \mathrm{ml})$ and the end of the donor is tied with the egg biomembrane. This donor compartment was immersed in the receiver compartment having $13 \mathrm{ml}$ of $\mathrm{pH} 7.4$ phosphate buffer solution. Sampling was done at different regular time interval for $36 \mathrm{hr}$. The samples were withdrawn completely and replaced with the fresh phosphate buffer solutions after every sampling. The samples were analyzed by UV Spectrophotometer for determining the released amount of the drug. The graph was plotted between the \%CDR and time. The other parameters like $r^{2}, \mathrm{t} 50$ and $\mathrm{t} 80 \%$ were calculated for evaluation of release study from different formulations and selection of best formulation.

\section{Stability Study}

The stability study was performed as per ICH guidelines. The formulated bionanosuspension were stored at different ambient temperatures for six months. The bionanosuspension were kept at two different conditions at $25^{\circ} \mathrm{C} \pm 2^{\circ} \mathrm{C}, 60 \% \mathrm{RH}$ and $40^{\circ} \mathrm{C} \pm 2^{\circ} \mathrm{C}, 75 \mathrm{RH}$ stability chamber. The samples were observed every two weeks for their different parameters during testing period. The samples under evaluation were observed for the drug content, $\mathrm{pH}$ changes and also any changes in color, appearance, its entrapment efficacy.

\section{RESULTS}

\section{Isolation of Biopolymer}

The Fragaria ananassa biopolymer was found to be light brown in color with \%yield of $13 \pm 2 \%$. The color changing point was found to be $229^{\circ} \mathrm{C} \pm 5^{\circ} \mathrm{C}$.

\section{Characterization of Isolated Biopolymer of Fragaria ananassa}

The isolated biopolymer was light brown in appearance. The biopolymer was found to be odorless with characteristic taste. It was found to be sparingly soluble in water. It showed the positive test for carbohydrate and protein. The Characterization of Isolated biopolymer of Fragaria ananassa is shown in Table 2.

\section{SEM analysis of biopolymer}

The isolated biopolymer was analyzed by scanning electron microscopy for surface characterization. The SEM analysis shows the rough and flaky structure of the biopolymer. Granular structure was also observed in SEM image. This confirms its polymeric nature of the biopolymer having flaky and granular structure. The SEM image of isolated biopolymer is shown in Figure 1.

\section{Different Spectral Analysis and their Findings} I.R. Spectral analysis of isolated biopolymer

FTIR spectra of the isolated biopolymer shows the presence of different functional groups which are responsible for the polymeric nature of the isolated biopolymer. The IR spectra shows the presence of different functional groups like hydroxyl $\left(3396.15 \mathrm{~cm}^{-1}\right)$, alkynes $\left(669.43 \mathrm{~cm}^{-}\right.$ $\left.{ }^{1}\right)$, carboxylic acid $\left(1410.35 \mathrm{~cm}^{-1}\right)$ which confirms its polymeric characteristics. The other groups like amide at $1639.02 \mathrm{~cm}^{-1}$, alkane at $2925.45 \mathrm{~cm}^{-1}$, tertiary alcohol at $1215.89 \mathrm{~cm}^{-1}$, were found to be present in the IR spectra. Presence of these functional groups is responsible for the retardibility in drug release like other standard polymer. IR spectra is shown in Figure 2a.

\section{Differential scanning colorimetry (DSC) Study of isolated biopolymer}

The DSC thermogram of Fragaria ananassa shows peaks at $83.794 \mathrm{Cel}$, $161.035 \mathrm{Cel}$ and $208.709 \mathrm{Cel}$. The area was found to be $229 \mathrm{mj} / \mathrm{mg}$, $30.5 \mathrm{mj} / \mathrm{mg}$ and $10.3 \mathrm{mj} / \mathrm{mg}$ respectively. The peak sshow the sharp endothermic peak broad peak which delas about the amorphous nature of biopolymer. Figure $2 \mathrm{~b}$.

\section{Mass spectroscopy of isolated biopolymer}

Mass spectra reveals that the isolated biopolymer is polymeric in nature due to presence of large molecular weight structure. It indicates the presence of protein. HRMS spectra of isolated biopolymer showed the parent peak at $\mathrm{m} / \mathrm{z} 456.33$ which confirms its large molecular weight structure like polymer. ${ }^{8}$ Figure $2 \mathrm{c}$.

\section{NMR spectroscopy of isolated biopolymer}

The NMR spectra show the presence of different peaks like multiplet at 0.829-0.902ppm which reveals the presence of primary alkyl group, peaks at $1.232 \mathrm{ppm}$ confirms the presence of methylene group, at $1.255 \mathrm{ppm}$ shows the presence of hydroxyl group. The presence of these groups confirms its polymeric nature. Figure $2 \mathrm{~d}$.

\section{Nanosizing of Phenytoin}

During the nanosizing of phenytoin after each sonication cycle the sample was observed for \%transmittance that confirm that as the number of cycle increases the $\%$ transmittance was increased. This was due to decrease in particle size and particles are now are in nanorange. Thus $\%$ transmittance shows the $\%$ of particles below $400 \mathrm{~nm}$ in bionanosuspension and \% blockade give an idea about the \% of particles which are above 400nm. Thus the UV method has given an idea about particles in nanorange.

\section{Drug-Excipient Interaction Study}

There was no any change in $\lambda_{\max }$ before $(216 \mathrm{~nm})$ and after the test $(216 \mathrm{~nm})$ in drug excipient study. The absorption maxima of phenytoinFragaria ananassa mixture was found to be closed to the $\lambda_{\max }$ of pure drug. There was no significant change in $\lambda_{\max }$ of drug-polymer mixture as compared to pure drug. It means it confirms that there was no any interaction between drug and biopolymer and other excipients also. It was observed that excipients is not interacting and not producing any changes in drug properties so the isolated biopolymer can be used for the preparation of bionanosuspension.

\section{Formulation of Phenytoin Loaded Bionanosuspension}

The different formulations of bionanoparticles by using different ratio of biopolymer from Fragaria ananassa and phenytoin were prepared. Then after formulation of bionanosuspension was evaluated for different parameters and their finding are described below.

\section{Dispensability Study of Bionanosuspension}

The dispersibility of the formulated bionanoparticles was found to be excellent. The redispersion was also found to be good. All nanoparticles 
Table 1: Formulation table of Phenytoin loaded bionanosuspension using Fragaria ananassa Biopolymer.

\begin{tabular}{cccccccccc}
\hline Formulations & PF1 & PF2 & PF3 & PF4 & PF5 & PF6 & PF7 & PF8 \\
\hline $\begin{array}{c}\text { Drug : Biopolymer ratio } \\
\text { Phenytoin }(\mathrm{mg})\end{array}$ & $1: 0.8$ & $1: 1$ & $1: 2$ & $1: 3$ & $1: 4$ & $1: 5$ & $1: 8$ & $1: 10$ \\
$\begin{array}{c}\text { Fragaria } \times \text { ananassa } \\
\text { (Biopolymer) }(\mathrm{mg})\end{array}$ & 10 & 10 & 10 & 10 & 10 & 10 & 10 & 10 \\
$\quad$ Polyvinyl alcohol $(\mathrm{ml})$ & 8 & 10 & 20 & 30 & 40 & 50 & 80 & 100 \\
$\quad$ Sodium benzoate (\%) & 0.5 & 0.5 & 0.5 & 0.5 & 0.5 & 0.5 & 0.5 & 0.5 \\
Double distilled Water $(\mathrm{ml})$ & 0.5 & 0.5 & 0.5 & 0.5 & 0.5 & 0.5 & 0.5 & 0.5 \\
& 10 & 10 & 10 & 10 & 10 & 10 & 10 & 10 \\
\hline
\end{tabular}

\section{Table 2: Characterization of Isolated Biopolymer of Fragaria ananassa.}

\begin{tabular}{cc}
\hline Parameters evaluated & Observation \\
\hline Color & Light brown \\
Odor & Characteristic \\
Taste & Characteristic \\
Melting Point & $229^{\circ} \mathrm{C} \pm 5^{\circ} \mathrm{C}$ \\
Solubility & Soluble in water, \\
& soluble in methanol \\
Carbohydrate & Present \\
Protein & Present \\
\hline
\end{tabular}

M.P. is expressed as mean $\pm \operatorname{SD}(n=3)$

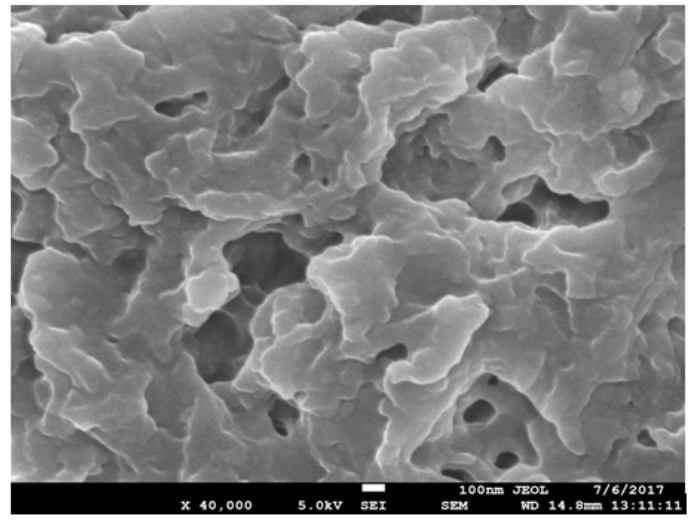

Figure 1: SEM of isolated biopolymer of biopolymer from Fragaria ananassa at $40,000 X$.

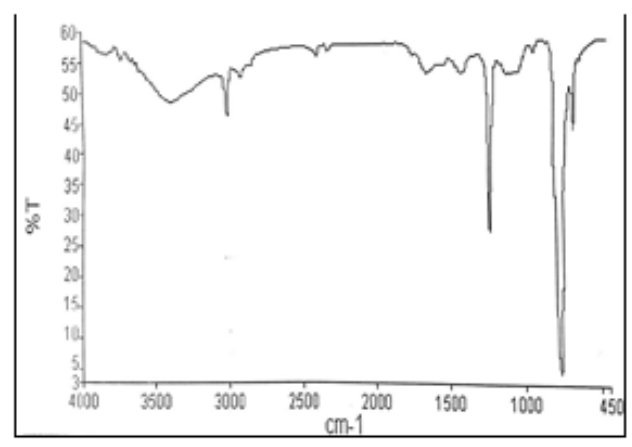

$2 \mathrm{a}$

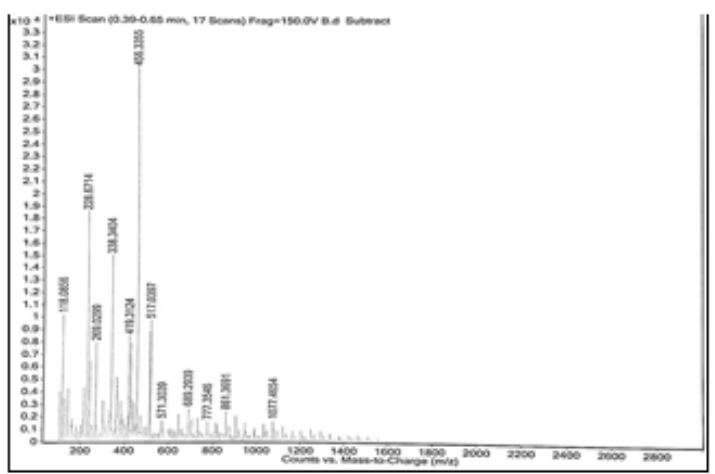

2c.

Figure 2: a. FT IR Spectra of biopolymer, 2. b. DSC of biopolymer, 2. c. High resolution Mass Spectrum of biopolymer, 2. d. NMR Spectra of biopolymer.

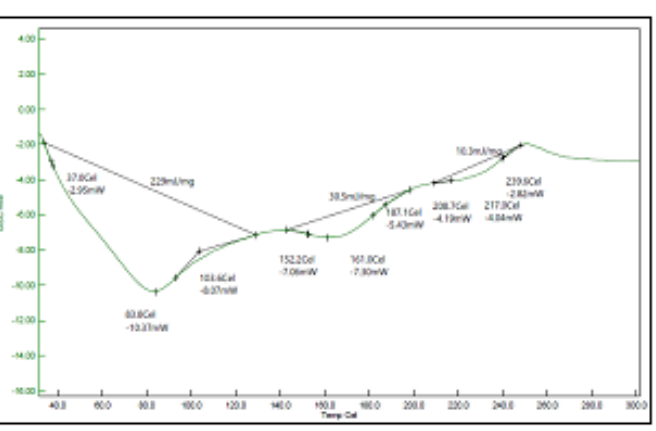

$2 \mathbf{b}$

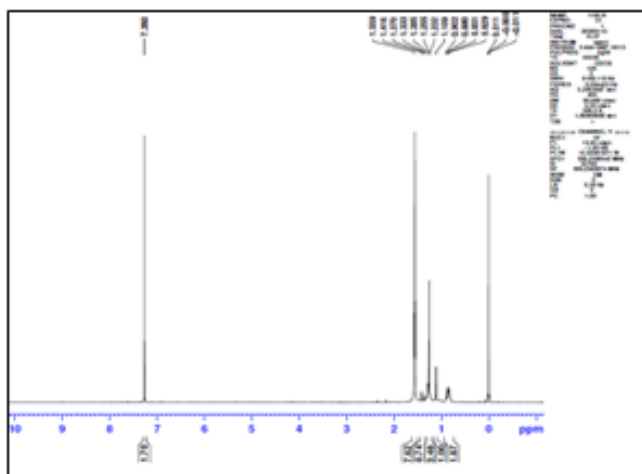

2d. 
were in dispersed state during dispersion. No aggregation or lump formation was observed.

\section{pH study of Bionanosuspension}

The $\mathrm{pH}$ of the bionanosuspension was found to be in range of $\mathrm{pH}$ $7.2 \pm 0.09$ to $\mathrm{pH} 7.7 \pm 0.19$. This means the formulations were in desired $\mathrm{pH}$ range that is suitable for the stability of the bionanosuspension. The $\mathrm{pH}$ of different bionanosuspension formulation observed is given in Table 3.

\section{\% Entrapment efficacy of loaded bionanoparticles}

The entrapment efficacy of the formulated bionanosuspension was found to be $81.88 \%$ to $89.69 \%$. Thus the formulated bionanosuspension showed the maximum entrapment efficacy up to $89.69 \%$.

\section{Evaluation \%transmittance of Bionanosuspension by UV method}

Here UV method has been used for screening the nanoparticles size in bionanosuspension. As the sonication cycle was increased the \% transmittance was found to be increased because the particle size after sonication has come in nanorange. The $\%$ transmittance indicated about the $\%$ of particles below $400 \mathrm{~nm}$ and the $\%$ blockade showed the $\%$ of particles above the $400 \mathrm{~nm}$ when screened by UV spectrophotometry method. Thus UV method can be used as a screening method for the evaluation of nanoparticles size in bionanosuspension. Thus nanosizing of Phenytoin and its utilization in preparation of bionanoparticles, nano particle size range may be easily screened by U.V. method. The screening

Table 3: Different formulations with observed pH values.

\begin{tabular}{cc}
\hline Formulations & Observed $\mathrm{pH}$ \\
\hline PF1 & $7.3 \pm 0.66$ \\
PF2 & $7.2 \pm 0.09$ \\
PF3 & $7.4 \pm 0.14$ \\
PF4 & $7.4 \pm 0.05$ \\
PF5 & $7.4 \pm 0.32$ \\
PF6 & $7.3 \pm 0.22$ \\
PF7 & $7.5 \pm 0.11$ \\
PF8 & $7.7 \pm 0.19$ \\
\hline
\end{tabular}

Results are expressed as mean $\pm \operatorname{SD}(n=3)$

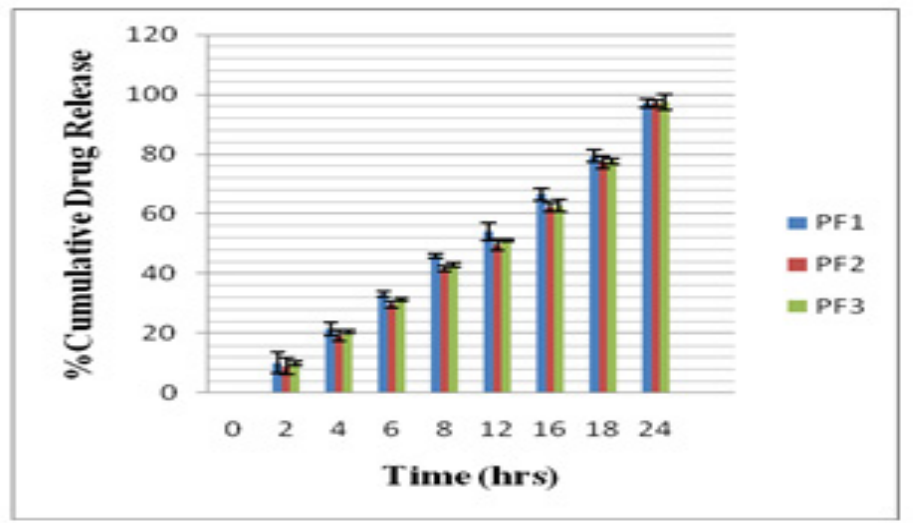

a of the bionanoparticles may be done in the same way as the nanosizing of phenytoin screening. Thus the UV method has given an idea about particles in nanorange.

\section{Particle size analysis}

The nanoparticles size in bionanosuspension (PF4) was found to be $615 \mathrm{~nm}$ after evaluation with Malvern Zetasizer. Thus the obtained size with the zeta potential of $-20.1 \mathrm{mV}$ confirms that the nanoparticles are in nanorange which is responsible for the stability of nanosuspension. It also confirms that the stable bionanosuspension loaded with phenytoin was prepared by using smart isolated Fragaria ananassa biopolymers. The result revels that it can be safely used for delivery of phenytoin from the prepared bionanoparticles in treatment of epilepsy. The Particle size distribution in bionanosuspension is shown in Figure 3.

\section{In vitro release study of bio-nanosuspension}

The in vitro release study was done by using modified M.S. diffusion apparatus. The release kinetic study was done by using the BIT-SOFT 1.12 software and $\mathrm{t} 50 \%$ and $\mathrm{t} 80 \%, \mathrm{r} 2$ were calculated. All the formulation showed more than $90.63 \%$ drug release (Figure $4 \mathrm{a}$ and $\mathrm{b}$ ). The in vitro release study of different formulations showed the \% drug release from $90.63 \%$ to $97.42 \%$. The different formulations were evaluated for the in vitro release study and release kinetic was studied. The formulation PF4 was found to be the best formulation having t50\% of $16.96 \mathrm{hr}$ and $\mathrm{t} 80 \%$ of $28 \mathrm{hr}$ with $r^{2}$ value of 0.9912 . The best formulation PF4 showed up to $90.63 \%$ drug release over $36 \mathrm{hr}$. According to the release kinetic study the

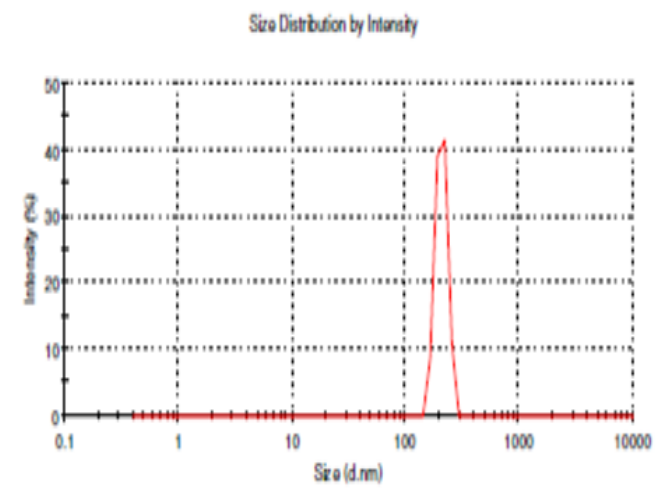

Figure 3: Particle size distribution in bionanosuspension.

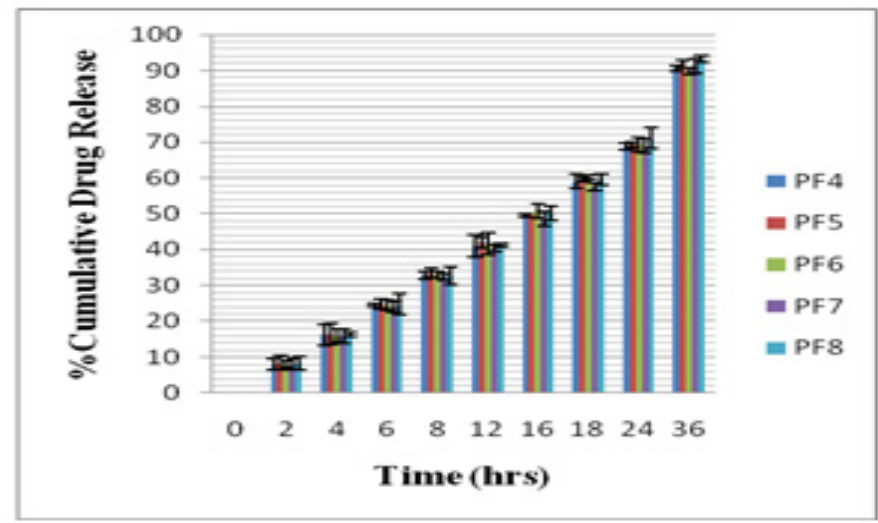

b

Figure 4: In vitro release drug profile of different bionanosuspension formulations (a.PF1-PF3 and b. PF4-PF8). The results are expressed as mean \pm SD ( $n=3$ ). 
best fit model was found to be Korsmeyer-Peppas and the mechanism of drug release was found to be anomalous transport. The result obtained from in vitro release study and analysis of the release kinetic of the all formulations indicates the sustained release of the phenytoin from the bionanosuspension.

\section{Stability Study}

The optimized formulations showed no any change in $\lambda_{\max }$, entrapment efficacy and in drug release. So there was no drug loss during the study period. The other evaluation parameters also showed the satisfactory result. The best optimized formulation was found to be stable over 6 months. There was no change in color, odor, $\mathrm{pH}$ and physical appearance. During the stability study period all the results obtained from different parameters were satisfactory and the formulation PF4 was found to be the best optimized stable formulation. During the study obtained results confirmed that the formulation was physically and chemically stable.

\section{DISCUSSION}

The biopolymer is a novel biomaterial which has a number of in-built properties which may be used for delivery of drugs to the target. The isolated biopolymer may be used for preparation of novel and intelligent carrier system for loading of phenytoin for treatment of epilepsy. The bionanoparticles are the nano range particulate systems which may be efficiently used for delivery of antiepileptic drug like phenytoin.

In this research work phenytoin was nanosized by novel and standard method. The biopolymer was isolated from the fruit of Fragaria ananassa has good polymeric properties. It can be suitably used for the preparation of bionanoparticles in the form of bionanosuspension. The isolated biopolymers showed good entrapment efficacy. This biopolymer has novel in-built properties like filmability, retardability and release rate controlling capability. It may be used for preparing suitable bionanosuspension for the delivery of nanosized phenytoin. In vitro release and release kinetic study reveals that the isolated Fragaria ananassa biopolymer consist of the desired bioretardant and biostabilizer novel properties. The nanosized phenytoin particle size was screened by UV method which gave an idea about the particle size range. This method may be used for screening of the nano size range of phenytoin as well as prepared bionanosuspension. ${ }^{9}$

The obtained results reveals that the isolated biopolymer consist of promising polymeric properties which can be used as an bioretaradant cum stabilizer for the preparation of stabilized bionanosuspension. The spectral characterization reveals about its polymeric nature.

The $\mathrm{pH}$, dispersibility and entrapment efficiency was found to be significant. The nanosizing of the drug showed the satisfactory results for entrapment as well as in overcoming the solubility problem of phenytoin.

The biopolymer showed the significant \% entrapment efficacy. From the values obtained it can be revealed excellent entrapment efficacy was achieved form the maximum of the bionanosuspension prepared using different biopolymers. So it was observed that the drug entrapment value increases with the increase in concentration of biopolymer. The isolated biopolymer showed good compatibility with the nanosized drugs.

The $\%$ transmittance measurement revealed that the prepared bionanosuspension have satisfactory particle size in nano range which is responsible for it stability.

The particle size of the formulated bionanosuspension was evaluated by measuring the $\%$ transmittance as well as with the help of measuring zeta particle size as well as zeta potential. ${ }^{7}$ The particle size for the zetasizer shows the particle size of $615 \mathrm{~nm}$ which confirms it nano particle size range for the best formulation PF4. The stability of bionanosuspension was also showed significant with $-20.1 \mathrm{mV}$ zeta potential. This means the particles are $\mathrm{n}$ well dispersed state without any agglomeration with good repulsive force.

In case for formulated bionanosuspension PF1 to PF8 by using the biopolymer Fragaria ananassa, it was observed that more than $90.63 \%$ drug was found to be released during study period. The formulation PF1-PF3 showed the burst release up to $97.416 \%$ in $24 \mathrm{hr}$. The bionanosuspension PF4-PF8 showed the controlled release of the drug. The bionanosuspension PF4 released the minimum drug as compared to other formulations in $36 \mathrm{hrs}$. On the basis of $\mathrm{t} 50 \%$ value it was observed that in release profile (Figure 5.124) the t50\%valuse was found to be $11.17 \mathrm{hr}$ for PF1 as minimum and $16.96 \mathrm{hrs}$ for PF4 as maximum. The formulation PF4 was selected as the best formulation as it showed the controlled drug release with $\mathrm{t} 80 \% 28.92 \mathrm{hrs}$.

Thus bionanosuspension (PF4) prepared by using the biopolymer from Fragaria ananassa showed the significant entrapment efficacy and sustained release of phenytoin for more than $36 \mathrm{hr}$. So biopolymer from Fragaria ananassa can be safely used for the formulation of stable bionanosuspension. ${ }^{10}$ The isolated Fragaria ananassa biopolymer was found to be novel, non-toxic, non-reactive, biocompatible, inert and biodegradable and effectively can be used in delivery of phenytoin for treatment of epilepsy. ${ }^{11}$ So the biopolymer can be safely used as the novel biomaterial in delivery of nanosized phenytoin. ${ }^{12}$ It can be safely used as an alternative to synthetic and semisynthetic available polymers for delivery of phenytoin in treatment of epilepsy. ${ }^{13}$

\section{CONCLUSION}

The research work concludes that the isolated biopolymer from Fragaria ananassa fruit serve as a potential, natural polymeric nature biomaterial which can be used for the preparation of nanosized phenytoin loaded bionanoparticles in the form of bionanosuspension. The best formulation PF4 prepared by using this natural and biodegradable isolated biopolymer from the edible natural fruit pulp was found to be stable. It releases the nanosized phenytoin in significant amount for the treatment of epilepsy. So it was concluded that the isolated biopolymer from Fragaria ananassa fruit may be safely used as the novel biomaterial in delivery of nanosized phenytoin from the formulated bionanoparticles in significant amount for extended time.

\section{ACKNOWLEDGEMENT}

I wish to acknowledge Prof. Devender Pathak (Dean, Faculty of Pharmacy, UPUMS, Saifai), Swarnima Pandey (Assistant Professor, AKTU, Lucknow, for encouraging me for the completion of research work. I want to also thank to SAIF, CDRI, Lucknow for providing me analytical testing facilities.

\section{CONFLICT OF INTEREST}

The author has no conflict of interest.

\section{ABBREVIATIONS}

Mg: Milligram; Nm: Nanometre; PF: Phenytoin loaded bionanoparticles; Ml: Millilitre; M.P.: Melting point.

\section{REFERENCES}

1. Baichwal MR. Polymer films as drug delivery systems. In: Advances in drug delivery systems. Bombay MSR Found. 1985;136-147.

2. Madhav NVS, Shankar MSU. A novel smart mucoadhesive biomaterial from Lallimantia royalena seed coat. J Science Asia. 2011;37:69-71.

3. Madhav NVS, Singh K. Smart ungual bio penetrant from the roots of Beta vulgaris. J Appl Pharm Sci. 2017;29:5(2):21-6

4. Madhav NVS, Tangri P. Formulation and evaluation of zidovudine bio-micro dwarfs using a novel bio muco-resident from Artocarpus heterophyllus. Int J 
Pharm Tech Res. 2011;3(1):169-174

5. Madhav NVS, Yadav AP. Development and evaluation of novel repaglinide biostrips for translabial delivery. Int Res J Pharm. 2013;4:198-202.

6. TyagiY, Madhav NVS. Design selegiline loaded bio-nanosuspension for the management of depression using novel bio-retardent from Manilkara zapota. Drug Dev. Ind. Pharm. 2019;45(8):1351-60.

7. Madhav NVS, Raina D. Formulation and evaluation of duloxetine loaded bionanosuspension for brain specificity via acoustic meatus. SOJ Pharmacy and Pharm Sci. 2017;4(1):1-5

8. Madhav NVS, Yadav AP. A novel Tran's labial platform utilizing bio excipients from Litchi chinensis for the delivery of rosiglitazone maleate. Acta Pharm Sin B. 2013:3(6):408-15.
9. Muller. Nanosuspensions as particulate drug formulation in therapy rationale for development and what we can expect for the future. Adv Drug Delivery. 2001;47(1):3-19.

10. Ojha A, Madhav NVS. A novel potent muco- bioadhesant polymer from seeds of Ricinus communis. World J Pharm Pharmaceut Sci. 2014;3:2154-65.

11. Patsalos PN, Froscher W, Pisani F. The importance of drug interactions in epilepsy therapy. Epilepsia. 2002;43(4):365-85.

12. Phillips MA, Gran ML, Peppas NA. Targeted Nano delivery of drugs and diagnostics. Nano Today. 2010;5(2):143-59.

13. Nilani P. Formulation and Evaluation of Polysaccharide Based Biopolymer-an Ecofriendly Alternative for Synthetic Polymer. J Pharm Sci Res. 2010;2(3):17884.

Article History: Submission Date : 19-03-2020; Revised Date : 04-06-2020; Acceptance Date : 22-07-2020.

Cite this article: Kumar S, Madhav NVS, Verma A, Pathak K. A Smart Approach for Delivery of Nanosized Phenytoin using Biomaterial Isolated from Fragaria ananassa. Int. J. Pharm. Investigation, 2020;10(3):305-11 